DOI: https://doi.org/10.24297/jam.v17i0.8481

\title{
A Parabolic Transform and Averaging Methods for General Partial Differential Equations
}

\author{
Mahmoud M. El-Borai \\ Faculty of Science, Alexandria University, Alexandria, Egypt \\ m m elborai@yahoo.com \\ Hamed Kamal Awad and Randa Hamdy. M. Ali \\ Faculty of Science, Damanhour University, Behera, Egypt \\ hamedk66@sci.dmu.edu.eg, rhamdy1989@gmail.com
}

\begin{abstract}
Averaging method of the fractional partial differential equations and a special case of these equations are studied, without any restrictions on the characteristic forms of the partial differential operators. We use the parabolic transform, existence and stability results can be obtained.
\end{abstract}

Keywords: Averaging method, fractional partial differential equation, parabolic transform, existence and uniqueness of solutions.

AMS Subject Classification 2010: 34 A 07, 34 A 60, 35 A 05, 26 A 33, 03 E 72, 34 C 29, 34 K 05, 34 K 30.

\section{Introduction}

Consider the following fractional partial differential equations:

$$
\begin{gathered}
\frac{\partial^{\alpha} u(x, t)}{\partial t^{\alpha}}=\varepsilon L(x, t, D) u(x, t), \\
u(x, 0)=\varphi(x),
\end{gathered}
$$

where

$$
L(x, t, D)=\sum_{|q| \leq m} a_{q}(x, t) D^{q},
$$

where $0<\alpha \leq 1, \varepsilon>0, D^{q}=D_{1}^{q_{1}} \ldots D_{n}^{q_{n}}, D_{j}=\frac{\partial}{\partial x_{j}}, j=1, \ldots, n, q=\left(q_{1}, \ldots, q_{n}\right)$ is an n-dimensional multi index, $|q|=q_{1}+\ldots+q_{n}, x=\left(x_{1}, \ldots, x_{n}\right) \in \Re^{n}, \Re^{n}$ is the $n$-dimensional Euclidean space, $0<t<T$.

Let $C_{b}\left(\Re^{n}\right)$ be the set of all bounded continuous functions on $\Re^{n}$. Consider the following Cauchy problem [8]:

$$
\frac{\partial u(x, t)}{\partial t}=\left(D_{1}^{2}+\ldots+D_{n}^{2}\right)^{2 N+1} u(x, t)
$$




$$
u(x, 0)=\varphi(x) \in C\left(\Re^{n}\right),
$$

where $C\left(\Re^{n}\right)$ is the set of all continuous functions on $\Re^{n}, N$ is a sufficiently large positive integer.

The solution of the Cauchy problem (3), (4) is given by

$$
u(x, t)=\int_{\Re^{n}} G(x-y, t) \varphi(y) d y,
$$

where $d y=d y_{1} \ldots d y_{n}$ and the function $G$ is the fundamental solution of the Cauchy problem (3), (4).

For sufficiently large $N$, we find $\gamma \in(0,1)$ and a constant $M>0$ such that:

$$
\max _{x}\left|D^{q} u(x, t)\right| \leq \frac{M}{t^{\gamma}} \max _{x}|\varphi(x)|,
$$

for all $t>0,|q| \leq m$.

A parabolic transform of a function $W$ is a function $\tilde{W}$ defined by [8]

$$
\tilde{W}\left(x, t_{1}, \ldots, t_{r}, c_{1} t+c_{2}\right)=\int_{\Re^{n}} G\left(x-y, c_{1} t+c_{2}\right) W\left(y, t_{1}, \ldots, t_{r}\right) d y,
$$

where $c_{1} \geq 0, c_{2} \geq 0, t_{j}, t \in[0, T], j=1, \ldots, r$ and $W\left(y, t_{1}, \ldots, t_{r}\right)$ is a continuous bounded function on $\Re^{n} \times[0, T]^{\gamma}$. By using the equations (1), (2), we get

$$
u(x, t)=\varphi(x)+\frac{\varepsilon}{\Gamma(\alpha)} \int_{0}^{t}(t-s)^{\alpha-1} L(x, s, D) u(x, s) d s,
$$

where $\Gamma$ is Gamma function.

In section 2, we study the averaging of the linear operator by using the parabolic transform where we generalize some known results due to Krol [3]. In section 3, we study a special case for the problem (1), (2) when $\alpha=1$. Compare $[1,2,4,5,6,7,8,9,10,11,13]$.

\section{Averaging a linear operator}

Consider the following equation [8]:

$$
v(x, t)=\tilde{\varphi}\left(x, c_{1} t\right)+\frac{\varepsilon}{\Gamma(\alpha)} \int_{0}^{t}(t-s)^{\alpha-1} \sum_{|q| \leq m} \tilde{a}_{q}\left(x, s, c_{1} t\right) D^{q} \tilde{v}\left(x, s, c_{2}-c_{1} s\right) d s
$$

where $c_{2} \geq c_{1} T, c_{1}, c_{2}$ are positive constants.

Let

$$
\tilde{L}\left(x, t, c_{1} t, D\right)=\sum_{|q| \leq m} \tilde{a}_{q}\left(x, t, c_{1} t\right) D^{q}
$$

and

$$
V(x, t)=\frac{\varepsilon}{\Gamma(\alpha)} \int_{0}^{t}(t-s)^{\alpha-1} \tilde{L}\left(x, s, c_{1} t, D\right) \tilde{v}\left(x, s, c_{2}-c_{1} s\right) d s
$$

we have

$$
\begin{gathered}
v(x, t)=\tilde{\varphi}\left(x, c_{1} t\right)+V(x, t) \\
\frac{\partial v(x, t)}{\partial t}=\frac{\partial \tilde{\varphi}\left(x, c_{1} t\right)}{\partial t}+\frac{\partial V(x, t)}{\partial t} \\
v(x, 0)=\tilde{\varphi}(x, 0)
\end{gathered}
$$


By averaging the coefficients $a_{q}(x, t), \tilde{a}_{q}\left(x, t, c_{1} t\right)$ over $t$, we can average the operator $L(x, t, D), \tilde{L}\left(x, t, c_{1} t, D\right)$,

$$
\begin{gathered}
\bar{a}_{q}(x)=\frac{1}{T} \int_{0}^{T} a_{q}(x, t) d t \\
\overline{\tilde{a}}_{q}(x)=\frac{1}{T} \int_{0}^{T} \tilde{a}_{q}\left(x, t, c_{1} t\right) d t
\end{gathered}
$$

for all $(x, t), x \in \Re^{n}$ producing the averaged operator $\bar{L}(x, D), \overline{\tilde{L}}(x, D)$, as an approximating problem for (1), (2) and (7) we take

$$
\begin{gathered}
\frac{\partial^{\alpha} u^{*}(x, t)}{\partial t^{\alpha}}=\varepsilon \bar{L}(x, D) u^{*}(x, t), \\
u^{*}(x, 0)=\varphi(x),
\end{gathered}
$$

and

$$
v^{*}(x, t)=\tilde{\varphi}\left(x, c_{1} t\right)+\frac{\varepsilon}{\Gamma(\alpha)} \int_{0}^{t}(t-s)^{\alpha-1} \overline{\tilde{L}}(x, D) \tilde{v}^{*}\left(x, s, c_{2}-c_{1} s\right) d s
$$

where

$$
\overline{\tilde{L}}(x, D)=\frac{1}{T} \int_{0}^{T} \sum_{|q| \leq m} \tilde{a}_{q}\left(x, t, c_{1} t\right) d t D^{q}
$$

let

$$
V^{*}(x, t)=\frac{\varepsilon}{\Gamma(\alpha)} \int_{0}^{t}(t-s)^{\alpha-1} \overline{\tilde{L}}(x, D) \tilde{v}^{*}\left(x, s, c_{2}-c_{1} s\right) d s,
$$

we have

$$
\begin{gathered}
v^{*}(x, t)=\tilde{\varphi}\left(x, c_{1} t\right)+V^{*}(x, t) \\
\frac{\partial v^{*}(x, t)}{\partial t}=\frac{\partial \tilde{\varphi}\left(x, c_{1} t\right)}{\partial t}+\frac{\partial V^{*}(x, t)}{\partial t}, \\
v^{*}(x, 0)=\tilde{\varphi}(x, 0) .
\end{gathered}
$$

By using the equations (15), (16), we get

$$
u^{*}(x, t)=\varphi(x)+\frac{\varepsilon}{\Gamma(\alpha)} \int_{0}^{t}(t-s)^{\alpha-1} \bar{L}(x, D) u^{*}(x, s) d s .
$$

Another straightforward analysis shows the existence and uniqueness of the solutions of the problems (1), (2), (7), (15), (16) and (17) on the time-scale $\frac{1}{\varepsilon}$.

The norm $\|.\|_{\infty}$ is defined by the supremum norm on the spatial domain and on the time-scale $\frac{1}{\varepsilon}$ and denoted by $\|u\|_{\infty}=\operatorname{Sup}_{x \in \Re^{n}}|u(x)|$.

Notice that there exist a dense set $E \in C_{b}\left(\Re^{n}\right)$.

Theorem 2.1 Let $u$ be the solution of the initial value problems (1), (2) and $u^{*}$ be the solution of the initial value problems (15), (16) then we have the estimate $\left\|u-u^{*}\right\|_{\infty}=O(\varepsilon)$ on the time-scale $\frac{1}{\varepsilon}$.

Proof. We introduce $\hat{v}(x, t)$ by the near-identity transformation:

$$
\hat{v}(x, t)=v^{*}(x, t)+\varepsilon \int_{0}^{t}\left(\tilde{L}\left(x, s, c_{1} t, D\right)-\overline{\tilde{L}}(x, D)\right) v^{*}(x, t) d s,
$$


and suppose that the derivatives of $v^{*}(x, t), \tilde{L}\left(x, t, c_{1} t, D\right)$ and $\overline{\tilde{L}}(x, D)$ are bounded.

If $n T \leq t \leq(n+1) T$ we obtain

$$
\begin{aligned}
\left\|\hat{v}(x, t)-v^{*}(x, t)\right\|_{\infty}= & \varepsilon\left\|\int_{n T}^{(n+1) T}\left(\tilde{L}\left(x, s, c_{1} t, D\right)-\overline{\tilde{L}}(x, D)\right) v^{*}(x, t) d s\right\|_{\infty} \\
= & \varepsilon \| \int_{n T}^{(n+1) T}\left[\sum_{|q| \leq m} \tilde{a}_{q}\left(x, s, c_{1} t\right) D^{q}\right. \\
& \left.-\frac{1}{T} \int_{0}^{T} \sum_{|q| \leq m} \tilde{a}_{q}\left(x, t, c_{1} t\right) d t D^{q}\right] v^{*}(x, t) d s \|_{\infty} \\
= & O(\varepsilon) \text { on the time-scale } \frac{1}{\varepsilon} .
\end{aligned}
$$

Differentiation of the near-identity transformation (24) and using the equations $(21,24)$ repeatedly, we have

$$
\begin{aligned}
& \frac{\partial \hat{v}(x, t)}{\partial t}=\frac{\partial v^{*}(x, t)}{\partial t}+\varepsilon\left(\tilde{L}\left(x, t, c_{1} t, D\right)-\overline{\tilde{L}}(x, D)\right) v^{*}(x, t) \\
& +\varepsilon \int_{0}^{t}\left(\tilde{L}\left(x, s, c_{1} t, D\right)-\overline{\tilde{L}}(x, D)\right) \frac{\partial v^{*}(x, t)}{\partial t} d s \\
& =\frac{\partial V^{*}(x, t)}{\partial t}+\frac{\partial \tilde{\varphi}\left(x, c_{1} t\right)}{\partial t}+\varepsilon\left(\tilde{L}\left(x, t, c_{1} t, D\right)-\overline{\tilde{L}}(x, D)\right) v^{*}(x, t) \\
& +\varepsilon \int_{0}^{t}\left(\tilde{L}\left(x, s, c_{1} t, D\right)-\overline{\tilde{L}}(x, D)\right)\left[\frac{\partial V^{*}(x, t)}{\partial t}+\frac{\partial \tilde{\varphi}\left(x, c_{1} t\right)}{\partial t}\right] d s \\
& =\varepsilon \tilde{L}\left(x, t, c_{1} t, D\right) v^{*}(x, t)+\frac{\partial V^{*}(x, t)}{\partial t}-\varepsilon \overline{\tilde{L}}(x, D) v^{*}(x, t) \\
& +\varepsilon \int_{0}^{t}\left(\tilde{L}\left(x, s, c_{1} t, D\right)-\overline{\tilde{L}}(x, D)\right) \frac{\partial V^{*}(x, t)}{\partial t} d s \\
& +\frac{\partial \tilde{\varphi}\left(x, c_{1} t\right)}{\partial t}+\varepsilon \int_{0}^{t}\left(\tilde{L}\left(x, s, c_{1} t, D\right)-\overline{\tilde{L}}(x, D)\right) \frac{\partial \tilde{\varphi}\left(x, c_{1} t\right)}{\partial t} d s \\
& =\varepsilon \tilde{L}\left(x, t, c_{1} t, D\right)\left[\hat{v}(x, t)-\varepsilon \int_{0}^{t}\left(\tilde{L}\left(x, s, c_{1} t, D\right)-\overline{\tilde{L}}(x, D)\right) v^{*}(x, t) d s\right] \\
& +\frac{\partial V^{*}(x, t)}{\partial t}-\varepsilon \overline{\tilde{L}}(x, D) v^{*}(x, t) \\
& +\varepsilon \int_{0}^{t}\left(\tilde{L}\left(x, s, c_{1} t, D\right)-\overline{\tilde{L}}(x, D)\right) \frac{\partial V^{*}(x, t)}{\partial t} d s \\
& +\frac{\partial \tilde{\varphi}\left(x, c_{1} t\right)}{\partial t}+\varepsilon \int_{0}^{t}\left(\tilde{L}\left(x, s, c_{1} t, D\right)-\overline{\tilde{L}}(x, D)\right) \frac{\partial \tilde{\varphi}\left(x, c_{1} t\right)}{\partial t} d s \\
& =\varepsilon \tilde{L}\left(x, t, c_{1} t, D\right) \hat{v}(x, t)+\frac{\partial V^{*}(x, t)}{\partial t}-\varepsilon \overline{\tilde{L}}(x, D) v^{*}(x, t) \\
& +\varepsilon\left[\int_{0}^{t}\left(\tilde{L}\left(x, s, c_{1} t, D\right)-\overline{\tilde{L}}(x, D)\right) \frac{\partial V^{*}(x, t)}{\partial t} d s\right. \\
& \left.-\varepsilon \tilde{L}\left(x, t, c_{1} t, D\right) \int_{0}^{t}\left(\tilde{L}\left(x, s, c_{1} t, D\right)-\overline{\tilde{L}}(x, D)\right) v^{*}(x, t) d s\right] \\
& +\frac{\partial \tilde{\varphi}\left(x, c_{1} t\right)}{\partial t}+\varepsilon \int_{0}^{t}\left(\tilde{L}\left(x, s, c_{1} t, D\right)-\overline{\tilde{L}}(x, D)\right) \frac{\partial \tilde{\varphi}\left(x, c_{1} t\right)}{\partial t} d s,
\end{aligned}
$$

with initial value $\hat{v}(x, 0)=\tilde{\varphi}(x, 0)$.

Let

$$
\frac{\partial}{\partial t}-\varepsilon \tilde{L}\left(x, t, c_{1} t, D\right)=\mathcal{L},
$$


we get

$$
\mathcal{L}\left(\hat{v}-v^{*}\right)=O(\varepsilon) \quad \text { on the time-scale } \frac{1}{\varepsilon}
$$

Moreover $\left(\hat{v}-v^{*}\right)(x, 0)=0, x \in \Re^{n}$.

To complete the proof we use the barrier functions and the Phragmèn-Lïndelöf principle see [12].

Suppose that the barrier function:

$$
\begin{aligned}
B(x, t) & =\varepsilon\|M(x, t)\|_{\infty} t \\
& +\| \frac{\partial V^{*}(x, t)}{\partial t}-\frac{\partial V(x, t)}{\partial t}+\varepsilon \tilde{L}\left(x, t, c_{1} t, D\right) v(x, t)-\varepsilon \overline{\tilde{L}}(x, D) v^{*}(x, t) \\
& +\varepsilon \int_{0}^{t}\left(\tilde{L}\left(x, s, c_{1} t, D\right)-\overline{\tilde{L}}(x, D)\right) \frac{\partial \tilde{\varphi}\left(x, c_{1} t\right)}{\partial t} d s \|_{\infty} t \\
& +\frac{1}{2} \varepsilon \| \tilde{L}\left(x, t, c_{1} t, D\right)\left[\frac{\partial V^{*}(x, t)}{\partial t}-\frac{\partial V(x, t)}{\partial t}\right. \\
& +\varepsilon \tilde{L}\left(x, t, c_{1} t, D\right) v(x, t)-\varepsilon \overline{\tilde{L}}(x, D) v^{*}(x, t) \\
& \left.+\varepsilon \int_{0}^{t}\left(\tilde{L}\left(x, s, c_{1} t, D\right)-\tilde{\tilde{L}}(x, D)\right) \frac{\partial \tilde{\varphi}\left(x, c_{1} t\right)}{\partial t} d s\right] \|_{\infty} t^{2},
\end{aligned}
$$

where

$$
\begin{aligned}
M(x, t)= & \int_{0}^{t}\left(\tilde{L}\left(x, s, c_{1} t, D\right)-\overline{\tilde{L}}(x, D)\right) \frac{\partial V^{*}(x, t)}{\partial t} d s \\
& -\varepsilon \tilde{L}\left(x, t, c_{1} t, D\right) \int_{0}^{t}\left(\tilde{L}\left(x, s, c_{1} t, D\right)-\overline{\tilde{L}}(x, D)\right) v^{*}(x, t) d s
\end{aligned}
$$

and the functions (we omit the arguments)

$$
Z_{1}(x, t)=\hat{v}(x, t)-v(x, t)-B(x, t), Z_{2}(x, t)=\hat{v}(x, t)-v(x, t)+B(x, t)
$$

We obtain

$$
\begin{aligned}
& \mathcal{L} Z_{1}(x, t)=\left(\frac{\partial}{\partial t}-\varepsilon \tilde{L}\left(x, t, c_{1} t, D\right)\right)[\hat{v}(x, t)-v(x, t)-B(x, t)] \\
& \quad=\frac{\partial V^{*}(x, t)}{\partial t}-\frac{\partial V(x, t)}{\partial t}+\varepsilon \tilde{L}\left(x, t, c_{1} t, D\right) v(x, t)-\varepsilon \overline{\tilde{L}}(x, D) v^{*}(x, t) \\
& \quad+\varepsilon \int_{0}^{t}\left(\tilde{L}\left(x, s, c_{1} t, D\right)-\overline{\tilde{L}}(x, D)\right) \frac{\partial \tilde{\varphi}\left(x, c_{1} t\right)}{\partial t} d s \\
& \quad+\varepsilon\left[\int_{0}^{t}\left(\tilde{L}\left(x, s, c_{1} t, D\right)-\tilde{\tilde{L}}(x, D)\right) \frac{\partial V^{*}(x, t)}{\partial t} d s\right. \\
& \left.\quad-\varepsilon \tilde{L}\left(x, t, c_{1} t, D\right) \int_{0}^{t}\left(\tilde{L}\left(x, s, c_{1} t, D\right)-\overline{\tilde{L}}(x, D)\right) v^{*}(x, t) d s\right] \\
& \quad-\varepsilon\|M(x, t)\|_{\infty}-\| \frac{\partial V^{*}(x, t)}{\partial t}-\frac{\partial V(x, t)}{\partial t}+\varepsilon \tilde{L}\left(x, t, c_{1} t, D\right) v(x, t)
\end{aligned}
$$




$$
\begin{aligned}
& -\varepsilon \overline{\tilde{L}}(x, D) v^{*}(x, t)+\varepsilon \int_{0}^{t}\left(\tilde{L}\left(x, s, c_{1} t, D\right)-\overline{\tilde{L}}(x, D)\right) \frac{\partial \tilde{\varphi}\left(x, c_{1} t\right)}{\partial t} d s \|_{\infty} \\
& -\varepsilon \| \tilde{L}\left(x, t, c_{1} t, D\right)\left[\frac{\partial V^{*}(x, t)}{\partial t}-\frac{\partial V(x, t)}{\partial t}+\varepsilon \tilde{L}\left(x, t, c_{1} t, D\right) v(x, t)\right. \\
& \left.-\varepsilon \overline{\tilde{L}}(x, D) v^{*}(x, t)+\varepsilon \int_{0}^{t}\left(\tilde{L}\left(x, s, c_{1} t, D\right)-\overline{\tilde{L}}(x, D)\right) \frac{\partial \tilde{\varphi}\left(x, c_{1} t\right)}{\partial t} d s\right] \|_{\infty} t \\
& +\varepsilon^{2} \tilde{L}\left(x, t, c_{1} t, D\right)\|M(x, t)\|_{\infty} t \\
& +\varepsilon \tilde{L}\left(x, t, c_{1} t, D\right) \| \frac{\partial V^{*}(x, t)}{\partial t}-\frac{\partial V(x, t)}{\partial t}+\varepsilon \tilde{L}\left(x, t, c_{1} t, D\right) v(x, t) \\
& -\varepsilon \overline{\tilde{L}}(x, D) v^{*}(x, t)+\varepsilon \int_{0}^{t}\left(\tilde{L}\left(x, s, c_{1} t, D\right)-\overline{\tilde{L}}(x, D)\right) \frac{\partial \tilde{\varphi}\left(x, c_{1} t\right)}{\partial t} d s \|_{\infty} t \\
& +\frac{1}{2} \varepsilon^{2} \tilde{L}\left(x, t, c_{1} t, D\right) \| \tilde{L}\left(x, t, c_{1} t, D\right)\left[\frac{\partial V^{*}(x, t)}{\partial t}-\frac{\partial V(x, t)}{\partial t}+\varepsilon \tilde{L}\left(x, t, c_{1} t, D\right) v(x, t)\right. \\
& \left.-\varepsilon \overline{\tilde{L}}(x, D) v^{*}(x, t)+\varepsilon \int_{0}^{t}\left(\tilde{L}\left(x, s, c_{1} t, D\right)-\overline{\tilde{L}}(x, D)\right) \frac{\partial \tilde{\varphi}\left(x, c_{1} t\right)}{\partial t} d s\right] \|_{\infty} t^{2} \\
& \leq 0
\end{aligned}
$$

$Z_{1}(x, 0)=0, x \in \Re^{n}$ similarly, $\mathcal{L} Z_{2}(x, t) \geq 0, Z_{2}(x, 0)=0, x \in \Re^{n}$.

$Z_{1}(x, t)$ and $Z_{2}(x, t)$ are bounded so we apply the Phragmèn-Lïndelöf principle, resulting in $Z_{1}(x, t) \leq 0$ and $Z_{2}(x, t) \geq 0$. We get

$$
-B(x, t) \leq \hat{v}(x, t)-v(x, t) \leq B(x, t)
$$

so we estimate

$$
\|\hat{v}(x, t)-v(x, t)\|_{\infty} \leq\|B(x, t)\|_{\infty}=O(\varepsilon)
$$

on the time-scale $\frac{1}{\varepsilon}$. We can use the triangle inequality to obtain

$$
\begin{aligned}
\left\|v(x, t)-v^{*}(x, t)\right\|_{\infty} & \leq\left\|\hat{v}(x, t)-v^{*}(x, t)\right\|_{\infty}+\|\hat{v}(x, t)-v(x, t)\|_{\infty} \\
& =O(\varepsilon) \text { on the time-scale } \frac{1}{\varepsilon} .
\end{aligned}
$$

If $\varphi, \bar{a}_{q} \in C_{b}\left(\Re^{n}\right), a_{q} \in C_{b}\left(\Re^{n} \times[0, T]\right)$, then $v\left(x, t, \frac{1}{n T}, \frac{1}{n}\right), v^{*}\left(x, t, \frac{1}{n T}, \frac{1}{n}\right)$ are the solutions of the equations (7), (17) with $c_{1}=\frac{1}{n T}$ and $c_{2}=\frac{1}{n}$.

Let

$$
\begin{aligned}
& u_{n}(x, t)=\int_{\Re^{n}} G\left(x-y, \frac{1}{n}-\frac{t}{n T}\right) v\left(y, t, \frac{1}{n T}, \frac{1}{n}\right) d y, \\
& u_{n}^{*}(x, t)=\int_{\Re^{n}} G\left(x-y, \frac{1}{n}-\frac{t}{n T}\right) v^{*}\left(y, t, \frac{1}{n T}, \frac{1}{n}\right) d y .
\end{aligned}
$$


By using the semi-group property and the equations (7), (17), we find that $u_{n}$ and $u_{n}^{*}$ satisfy the following equations

$$
\begin{gathered}
u_{n}(x, t)=\varphi_{n}(x)+\frac{\varepsilon}{\Gamma(\alpha)} \sum_{|q| \leq m} \int_{0}^{t}(t-s)^{\alpha-1} a_{q, n}(x, s) D^{q} u_{n}(x, s) d s, \\
u_{n}^{*}(x, t)=\varphi_{n}(x)+\frac{\varepsilon}{\Gamma(\alpha)} \sum_{|q| \leq m} \int_{0}^{t}(t-s)^{\alpha-1} \bar{a}_{q, n}(x) D^{q} u_{n}^{*}(x, s) d s,
\end{gathered}
$$

where $\varphi_{n}, a_{q, n}, \bar{a}_{q, n}$ are defined as in

$$
\begin{gathered}
v_{n+1}(x, t)=\tilde{\varphi}\left(x, c_{1} t\right)+\frac{\varepsilon}{\Gamma(\alpha)} \int_{0}^{t}(t-s)^{\alpha-1} \sum_{|q| \leq m} \tilde{a}_{q}\left(x, s, c_{1} t\right) D^{q} \tilde{v}_{n}\left(x, s, c_{2}-c_{1} s\right) d s, \\
v_{n+1}^{*}(x, t)=\tilde{\varphi}\left(x, c_{1} t\right)+\frac{\varepsilon}{\Gamma(\alpha)} \int_{0}^{t}(t-s)^{\alpha-1} \sum_{|q| \leq m} \overline{\tilde{a}}_{q}(x) D^{q} \tilde{v}_{n}^{*}\left(x, s, c_{2}-c_{1} s\right) d s
\end{gathered}
$$

where

$$
\tilde{v}_{n}\left(x, s, c_{2}-c_{1} s\right)=\int_{\Re^{n}} G\left(x-y, c_{2}-c_{1} s\right) v_{n}(y, s) d y,
$$

and

$$
\tilde{v}_{n}^{*}\left(x, s, c_{2}-c_{1} s\right)=\int_{\Re^{n}} G\left(x-y, c_{2}-c_{1} s\right) v_{n}^{*}(y, s) d y .
$$

Hence the required result.

Notice that if the coefficients $a_{q}$ 's and $\bar{a}_{q}$ 's do not depend on $x$ for all $|q| \leq m$, then $a_{q, n}=a_{q}$ and $\bar{a}_{q, n}=\bar{a}_{q}$.

\section{A special case}

We study a special case for problem (1), (2) when $\alpha=1$ :

$$
\begin{gathered}
\frac{\partial}{\partial t} u(x, t)=\varepsilon L(x, t, D) u(x, t), \\
u(x, 0)=\varphi(x) .
\end{gathered}
$$

We have

$$
u(x, t)=\varphi(x)+\varepsilon \int_{0}^{t} L(x, s, D) u(x, s) d s,
$$

let

$$
v(x, t)=\tilde{\varphi}\left(x, c_{1} t\right)+\varepsilon \int_{0}^{t} \sum_{|q| \leq m} \tilde{a}_{q}\left(x, s, c_{1} t\right) D^{q} \tilde{v}\left(x, s, c_{2}-c_{1} s\right) d s,
$$

and

$$
V_{1}(x, t)=\varepsilon \int_{0}^{t} \sum_{|q| \leq m} \tilde{a}_{q}\left(x, s, c_{1} t\right) D^{q} \tilde{v}\left(x, s, c_{2}-c_{1} s\right) d s
$$

we have

$$
\begin{gathered}
v(x, t)=\tilde{\varphi}\left(x, c_{1} t\right)+V_{1}(x, t), \\
\frac{\partial v(x, t)}{\partial t}=\frac{\partial \tilde{\varphi}\left(x, c_{1} t\right)}{\partial t}+\frac{\partial V_{1}(x, t)}{\partial t}, \\
v(x, 0)=\tilde{\varphi}(x, 0)
\end{gathered}
$$


By averaging the coefficients $a_{q}(x, t), \tilde{a}_{q}\left(x, t, c_{1} t\right)$ over $t$, we can average the operator $L(x, t, D), \tilde{L}\left(x, t, c_{1} t, D\right)$, for all $(x, t), x \in \Re^{n}$ producing the averaged operator $\bar{L}(x, D), \overline{\tilde{L}}(x, D)$, as an approximating problem for (26), (27) and (29) we take

$$
\begin{gathered}
\frac{\partial u^{*}(x, t)}{\partial t}=\varepsilon \bar{L}(x, D) u^{*}(x, t), \\
u^{*}(x, 0)=\varphi(x),
\end{gathered}
$$

and

$$
v^{*}(x, t)=\tilde{\varphi}\left(x, c_{1} t\right)+\varepsilon \int_{0}^{t} \overline{\tilde{L}}(x, D) \tilde{v}^{*}\left(x, s, c_{2}-c_{1} s\right) d s
$$

let

$$
V_{1}^{*}(x, t)=\varepsilon \int_{0}^{t} \overline{\tilde{L}}(x, D) \tilde{v}^{*}\left(x, s, c_{2}-c_{1} s\right) d s
$$

we get

$$
\begin{gathered}
v^{*}(x, t)=\tilde{\varphi}\left(x, c_{1} t\right)+V_{1}^{*}(x, t), \\
\frac{\partial v^{*}(x, t)}{\partial t}=\frac{\partial \tilde{\varphi}\left(x, c_{1} t\right)}{\partial t}+\frac{\partial V_{1}^{*}(x, t)}{\partial t}, \\
v^{*}(x, 0)=\tilde{\varphi}(x, 0) .
\end{gathered}
$$

From the equations(34), (35), we have

$$
u^{*}(x, t)=\varphi(x)+\varepsilon \int_{0}^{t} \bar{L}(x, D) u^{*}(x, s) d s,
$$

Theorem 3.2 Let $u$ be the solution of the initial value problems (26), (27) and $u^{*}$ be the solution of the initial value problems (34), (35) then we have the estimate $\left\|u-u^{*}\right\|_{\infty}=O(\varepsilon)$ on the time-scale $\frac{1}{\varepsilon}$.

Proof. Consider the near-identity transformation:

$$
\hat{v}(x, t)=v^{*}(x, t)+\varepsilon \int_{0}^{t}\left(\tilde{L}\left(x, s, c_{1} t, D\right)-\overline{\tilde{L}}(x, D)\right) v^{*}(x, t) d s,
$$

we obtain

$$
\left\|\hat{v}(x, t)-v^{*}(x, t)\right\|_{\infty}=O(\varepsilon) \quad \text { on the time-scale } \frac{1}{\varepsilon} .
$$

By differentiating of the near-identity transformation (42) and using the equations $(39,42)$ repeatedly, we get

$$
\begin{aligned}
\frac{\partial \hat{v}(x, t)}{\partial t}= & \varepsilon \tilde{L}\left(x, t, c_{1} t, D\right) \hat{v}(x, t)+\frac{\partial V_{1}^{*}(x, t)}{\partial t}-\varepsilon \overline{\tilde{L}}(x, D) v^{*}(x, t) \\
& +\varepsilon\left[\int_{0}^{t}\left(\tilde{L}\left(x, s, c_{1} t, D\right)-\overline{\tilde{L}}(x, D)\right) \frac{\partial V_{1}^{*}(x, t)}{\partial t} d s\right. \\
& \left.-\varepsilon \tilde{L}\left(x, t, c_{1} t, D\right) \int_{0}^{t}\left(\tilde{L}\left(x, s, c_{1} t, D\right)-\overline{\tilde{L}}(x, D)\right) v^{*}(x, t) d s\right] \\
& +\frac{\partial \tilde{\varphi}\left(x, c_{1} t\right)}{\partial t}+\varepsilon \int_{0}^{t}\left(\tilde{L}\left(x, s, c_{1} t, D\right)-\overline{\tilde{L}}(x, D)\right) \frac{\partial \tilde{\varphi}\left(x, c_{1} t\right)}{\partial t} d s,
\end{aligned}
$$

with initial value $\hat{v}(x, 0)=\tilde{\varphi}(x, 0)$.

We have 


$$
\mathcal{L}\left(\hat{v}-v^{*}\right)=O(\varepsilon) \quad \text { on the time-scale } \frac{1}{\varepsilon}
$$

Moreover $\left(\hat{v}-v^{*}\right)(x, 0)=0, x \in \Re^{n}$.

We can use the barrier functions and the Phragmèn-Lïndelöf principle see [12].

Let the barrier function:

$$
\begin{aligned}
B_{1}(x, t) & =\varepsilon\left\|M_{1}(x, t)\right\|_{\infty} t \\
& +\| \frac{\partial V_{1}^{*}(x, t)}{\partial t}-\frac{\partial V_{1}(x, t)}{\partial t}+\varepsilon \tilde{L}\left(x, t, c_{1} t, D\right) v(x, t)-\varepsilon \overline{\tilde{L}}(x, D) v^{*}(x, t) \\
& +\varepsilon \int_{0}^{t}\left(\tilde{L}\left(x, s, c_{1} t, D\right)-\overline{\tilde{L}}(x, D)\right) \frac{\partial \tilde{\varphi}\left(x, c_{1} t\right)}{\partial t} d s \|_{\infty} t \\
& +\frac{1}{2} \varepsilon \| \tilde{L}\left(x, t, c_{1} t, D\right)\left[\frac{\partial V_{1}^{*}(x, t)}{\partial t}-\frac{\partial V_{1}(x, t)}{\partial t}\right. \\
& +\varepsilon \tilde{L}\left(x, t, c_{1} t, D\right) v(x, t)-\varepsilon \overline{\tilde{L}}(x, D) v^{*}(x, t) \\
& \left.+\varepsilon \int_{0}^{t}\left(\tilde{L}\left(x, s, c_{1} t, D\right)-\tilde{\tilde{L}}(x, D)\right) \frac{\partial \tilde{\varphi}\left(x, c_{1} t\right)}{\partial t} d s\right] \|_{\infty} t^{2},
\end{aligned}
$$

where

$$
\begin{aligned}
M_{1}(x, t)= & \int_{0}^{t}\left(\tilde{L}\left(x, s, c_{1} t, D\right)-\overline{\tilde{L}}(x, D)\right) \frac{\partial V_{1}^{*}(x, t)}{\partial t} d s \\
& -\varepsilon \tilde{L}\left(x, t, c_{1} t, D\right) \int_{0}^{t}\left(\tilde{L}\left(x, s, c_{1} t, D\right)-\overline{\tilde{L}}(x, D)\right) v^{*}(x, t) d s
\end{aligned}
$$

and the functions (we omit the arguments)

$$
Z_{3}(x, t)=\hat{v}(x, t)-v(x, t)-B_{1}(x, t), Z_{4}(x, t)=\hat{v}(x, t)-v(x, t)+B_{1}(x, t)
$$

We have

$\mathcal{L} Z_{3}(x, t) \leq 0, Z_{3}(x, 0)=0$ similarly, $\mathcal{L} Z_{4}(x, t) \geq 0, Z_{4}(x, 0)=0$.

$Z_{3}(x, t)$ and $Z_{4}(x, t)$ are bounded so we apply the Phragmèn-Lïndelöf principle, resulting in $Z_{3}(x, t) \leq 0$ and $Z_{4}(x, t) \geq$ 0 . We get

$$
-B_{1}(x, t) \leq \hat{v}(x, t)-v(x, t) \leq B_{1}(x, t)
$$

so we estimate

$$
\|\hat{v}(x, t)-v(x, t)\|_{\infty} \leq\left\|B_{1}(x, t)\right\|_{\infty}=O(\varepsilon),
$$

on the time-scale $\frac{1}{\varepsilon}$. We can use the triangle inequality to get

$$
\left\|v(x, t)-v^{*}(x, t)\right\|_{\infty}=O(\varepsilon) \text { on the time-scale } \frac{1}{\varepsilon} .
$$

Similar to section (2), we have the required result.

\section{Conclusion}

A fractional partial differential equation can be solved without any restrictions on the characteristic forms by using the parabolic transform and the averaging methods. As a special case Cauchy problem is solved for a fractional partial differential equation. 


\section{References}

[1] A. Ben Lemlih and J.A. Ellison, Method of averaging and the quantum anharmonic oscillator, Phys. Rev. Lett. 55 (1985), 1950-1953.

[2] A. Friedman, Partial Differential Equations of Parabolic Type, Prentice-Hall, Englewood Cliffs, NJ (1964).

[3] Krol, M.S., On the averaging method in nearly time-periodic advection-diffusion problems, SIAM J. Appl. Math. 51 (1991), 1622-1637.

[4] Mahmoud M. El-Borai, Evolution equations without semi groups, J. of Appl. Math. And Comp., 149 (2004), 815-821.

[5] Mahmoud M. El-Borai, Some probability densities and fundamental solutions of fractional evolution equations, Chaos, Soliton and Fractals 14 (2002), 433-440.

[6] Mahmoud M. El-Borai, O. L. Moustafa, F. H. Michael, On the correct formulation of a nonlinear differential equations in Banach space, Int. J. Math. 22(1) (1999).

[7] Mahmoud M. El-Borai, Khairia El-Said El-Nadi, A parabolic transform and some stochastic Ill-posed problem, British Journal of Mathematics and Computer Science, 9(5) (2015), 418-426.

[8] Mahmoud M. El-Borai, Khairia El-Said El-Nadi, On the solutions of Ill-posed Cauchy problems for some singular integro-partial differential equations, Global Journal of Mathematics, 13(2) (2019), 899-905.

[9] Mahmoud M. El-Borai, Khairia El-Said El-Nadi and Eman G. El-Akabawy, On some fractional evolution equations, Computers and Mathematics with Applications, 59 (2010), 1352-1355.

[10] Mahmoud M. El-Borai, Khairia El-Said El-Nadi and Hoda A. Foad, On some fractional stochastic delay differential equations, Computers and Mathematics with Applications, 59 (2010), 1165-1170.

[11] Mahmoud M. El-Borai, Wagdy G. El-Sayed, Faez N. Ghaffoori, On the Cauchy problem for some parabolic fractional partial differential equations with time delays, Journal of Mathematics and System Science, 6 (2016), 194-199.

[12] Protter, M.H. and Weinberger, H.F., Maximum Principles in Differential Equations, Prentice-Hall, Englewood Cliffs, NJ (1967).

[13] Verhulst, F., On averaging methods for partial differential equations, SPT98-Symmetry and Perturbation Theory II, A.Degasperis and G.Gaeta eds., World Scientifc (1999), 79-95. 